\title{
A Three-Dimensional Spheroid Model to Investigate the Tumor-Stromal Interaction in Hepatocellular Carcinoma
}

\author{
Marco Y. W. Zaki ${ }^{*}, 1,2$, Shishir Shetty ${ }^{*}, 2$, Alex L. Wilkinson ${ }^{2}$, Daniel A. Patten ${ }^{2}$, Fiona Oakley ${ }^{*}, 3$, Helen Reeves ${ }^{*}, 4,5$ \\ ${ }^{1}$ Department of Biochemistry, Faculty of Pharmacy, Minia University ${ }^{2}$ National Institute for Health Research Birmingham Liver Biomedical Research Unit \\ and Centre for Liver and Gastrointestinal Research, Institute of Immunology and Immunotherapy, University of Birmingham ${ }^{3}$ Newcastle Fibrosis Research \\ Group, Biosciences Institute, Faculty of Medical Sciences, Newcastle University ${ }^{4}$ Newcastle University Translational and Clinical Research Institute, Faculty \\ of Medical Sciences, Newcastle University ${ }^{5}$ The Liver Unit, Department of Medicine, Freeman Hospital, Newcastle-upon-Tyne Hospitals NHS Foundation \\ Trust \\ "These authors contributed equally
}

\section{Corresponding Author}

Marco Y. W. Zaki

marcozaki@mu.edu.eg

\section{Citation}

Zaki, M.Y.W., Shetty, S., Wilkinson, A.L., Patten, D.A., Oakley, F., Reeves, H. A Three-Dimensional Spheroid Model to Investigate the TumorStromal Interaction in Hepatocellular Carcinoma. J. Vis. Exp. (175), e62868, doi:10.3791/62868 (2021).

\section{Date Published}

September 30, 2021

DOI

$10.3791 / 62868$

URL

jove.com/video/62868

\section{Abstract}

The aggressiveness and lack of well-tolerated and widely effective treatments for advanced hepatocellular carcinoma ( $\mathrm{HCC}$ ), the predominant form of liver cancer, rationalize its rank as the second most common cause of cancer-related death. Preclinical models need to be adapted to recapitulate the human conditions to select the best therapeutic candidates for clinical development and aid the delivery of personalized medicine. Three-dimensional (3D) cellular spheroid models show promise as an emerging in vitro alternative to two-dimensional (2D) monolayer cultures. Here, we describe a 3D tumor spheroid model which exploits the ability of individual cells to aggregate when maintained in hanging droplets, and is more representative of an in vivo environment than standard monolayers. Furthermore, 3D spheroids can be produced by combining homotypic or heterotypic cells, more reflective of the cellular heterogeneity in vivo, potentially enabling the study of environmental interactions that can influence progression and treatment responses. The current research optimized the cell density to form 3D homotypic and heterotypic tumor spheroids by immobilizing cell suspensions on the lids of standard $10 \mathrm{~cm}^{3}$ Petri dishes. Longitudinal analysis was performed to generate growth curves for homotypic versus heterotypic tumor/fibroblasts spheroids. Finally, the proliferative impact of fibroblasts (COS7 cells) and liver myofibroblasts (LX2) on homotypic tumor (Hep3B) spheroids was investigated. A seeding density of 3,000 cells (in $20 \mu \mathrm{L}$ media) successfully yielded Huh7/COS7 heterotypic spheroids, which displayed a steady increase in size up to culture day 8 , followed by growth retardation. This finding was corroborated using Hep3B homotypic spheroids cultured in LX2 (human hepatic stellate cell line) conditioned medium (CM). LX2 CM triggered the proliferation of 
Hep3B spheroids compared to control tumor spheroids. In conclusion, this protocol has shown that $3 D$ tumor spheroids can be used as a simple, economical, and prescreen in vitro tool to study tumor-stromal interactions more comprehensively.

\section{Introduction}

The global incidence and mortality from liver cancer have continued to increase, despite advances in treatments for liver disease and most other types of cancer. In 2018, liver cancer surpassed colorectal and stomach cancer to become the second most common cause of cancer-related death globally ${ }^{1}$. In 2020 , there were more than 9,00,000 new diagnoses, accounting for $4.7 \%$ of total cancer cases worldwide ${ }^{1}$. This is particularly disappointing, given that the significant risk factors for the development of $\mathrm{HCC}$, the most common form of liver cancer, are well characterised ${ }^{2}$. Cirrhosis is the most common risk factor for the development of $\mathrm{HCC}$, with $80 \%$ of cases developing on the background of established cirrhosis ${ }^{2}$. Chronic liver diseases, which progress to cirrhosis, and consequently HCC, include Hepatitis B virus (HBV), Hepatitis C virus (HCV), alcohol-related liver disease (ARLD), non-alcoholic fatty liver diseases (NAFLD) - the latter attributed to obesity and type 2 diabetes mellitus $(\mathrm{T} 2 \mathrm{DM})^{2,3}$. The current management protocols for $\mathrm{HCC}$ are stage-dependent and limited for those with advanced cancer, who most often have a poor outcome ${ }^{4}$. There have been significant advances using kinase inhibitors and, more recently, immune-oncology treatments, although realistically, benefit only a minority of patients with advanced liver cancers $^{5}$. Moreover, there is concern that HCCs arising in patients with NAFLD - the most rapidly growing underlying cause, accounting for more than $50 \%$ of newly diagnosed
HCC cases in western nations, may be more resistant to Programmed death 1 (PD1) checkpoint inhibitor therapy ${ }^{6}$.

There has been a massive investment in clinical trials for patients with HCC, including significant improvements in clinical trials and their endpoints ${ }^{7}$. After a decade of failures, these investments have started to change the opportunities for patients. However, the reality is that the overall proportion of responders remains relatively poor, with patients recruited into trials often poorly representing those cared for in the clinics. The danger is that the advances are costly and benefit the few rather than the many. As more candidate therapies emerge for single-use or combination, it is essential to have preclinical models more predictive of in vivo responses. These are likely to be models that incorporate additional factors contributing to the variability seen in patient responses that better reflect human HCC heterogeneity and pathological complexity ${ }^{8}$. Systems that recreate the in vivo pathophysiological conditions of $\mathrm{HCC}$ are needed to help understand the biology of tumor evolution, growth, and progression. The existing experimental models of chronic liver diseases and HCC usually fall under three main categories: in vivo animal-based models (reviewed $\mathrm{in}^{9}$ ), in vitro cultures ${ }^{10}$, and ex vivo ${ }^{11,12}$ models. Animalbased approaches are extensively used to study chronic liver diseases, including HCC; however, the genetic variability, high running costs, and the different immune systems between species are among the main limitations for applying such models ${ }^{9}$. While some ex vivo models provide an 
excellent tool for focusing on human tissues compared to other in vitro cell line models, tissue availability and the limited experimental time course obstruct their utilization on a large scale.

On the other hand, the in vitro cell line models remain a good option for scientists working with limited resources, with a lesser need to have a constant supply of fresh human tissues ${ }^{10}$. These models also provide a tool that can be used as a first screen to help with target validation of drug selection before proceeding to more complex in vivo models. The recent modification of the traditional $2 \mathrm{D}$ monolayer cultures into 3D cultures has improved the efficacy of these in vitro models $^{13,14}$.

3D in vitro models can recapitulate critical features seen in human normal and pathological conditions. Under physiological conditions, signal transduction is initiated through cellular crosstalk and interaction with other connective tissue molecules, namely, the extracellular matrix (ECM) proteins, forming a 3D interaction network ${ }^{15,16}$. The tumor evolves in a 3D spherical form during malignant transformation, for which oxygen and nutrients are readily abundant in non-tumor/tumor interphase. At the same time, hypoxic conditions predominate at the tumor core. This heterogeneity in nutrient availability results in the activation of spatially distinct signaling and metabolic pathways that regulate tumorigenesis. These conditions are poorly recapitulated in the conventional $2 \mathrm{D}$ monolayer cultures ${ }^{14}$, in which cells grow on stiff culture plastic in a physiologically irrelevant fashion. Cancer cells also communicate with other non-parenchymal cells, the primary source of ECM, growth, and invasion signaling within the tumor microenvironment. Unlike 2D cultures, 3D in vitro models can provide a more suitable platform to study this tumor-stromal interaction ${ }^{17}$.
3D models are widely used in the HCC field, and they vary in the way the micro-tissue is formed $18,19,20,21,22,23$. Most of these models used either the ultra-low binding plates $^{18,19,20,21,22}$ or trans-wells ${ }^{23}$ in the process of spheroid formation. The protocol described introduces the hanging droplet technique as an alternative, plastic-free, and cost-effective in vitro 3D tumor spheroid model. This may facilitate assessing the paracrine and autocrine roles of fibroblasts on the proliferation of the tumor cells in a $3 D$ format.

\section{Protocol}

\section{Cell preparation}

1. Perform all the experiments under sterile conditions in Class II laminar flow microbiological safety cabinet (see Table of Materials).

1. Turn on the hood and allow for the stabilization of the airflow.

2. Thoroughly spray the interior hood surface with $70 \%$ ethanol to eliminate any possible contamination from previous users.

3. Prepare $5 \%$ of a disinfectant solution in a $500 \mathrm{~mL}$ glass beaker. Discard any cell supernatant or cell debris inside the solution.

4. Thoroughly clean all the micropipettes and tip boxes with $70 \%$ ethanol.

2. Prepare fresh cell culture media by supplementing Dulbecco's modified Eagle's medium (DMEM) high glucose media with $10 \%$ heat deactivated fetal bovine serum (FBS), 1\% penicillin/streptomycin (100 unit/mL of penicillin and $100 \mu \mathrm{g} / \mathrm{mL}$ of streptomycin), and $2 \mathrm{mM} \mathrm{L-}$ 
glutamine. For the LX2 hepatic stellate cell line, reduce the concentration of the FBS supplement to $2 \%$.

3. Warm culture media before initiating the experiment.

4. Take Huh7 and Hep3B HCC tumor cell lines and COS7 and LX2 fibroblast cell lines from their storage rack in liquid nitrogen. Rapidly defrost cryopreserved cells.

5. Dilute thawed cells with $2 \mathrm{~mL}$ of fresh culture media. Centrifuge cells at $200 \times \mathrm{g}$ for $4 \mathrm{~min}$ at room temperature. Discard the supernatant and resuspend the cell pellet in $1 \mathrm{~mL}$ of fresh warm culture media.

6. Seed cells in T75 cell culture flask. Incubate the cells in a cell culture incubator in $5 \% \mathrm{CO}_{2}$ at $37{ }^{\circ} \mathrm{C}$ in 95\% humidified conditions until cells reach $60 \%-70 \%$ confluency.

\section{Cell collection}

1. Aspirate culture media and wash cells three times with phosphate buffer saline (PBS).

2. Add $2 \mathrm{~mL}$ of pre-warmed $1 \mathrm{x}$ Trypsin to detach adherent cells from the bottom of the $T 75$ flasks. Incubate at $37^{\circ} \mathrm{C}$ in an incubator for $4 \mathrm{~min}$.

3. Inactivate trypsin by adding $4 \mathrm{~mL}$ of complete culture media. Collect the cell suspension and centrifuge cells at $200 \times g$ for $4 \mathrm{~min}$ at room temperature. Discard the supernatant and resuspend the cells in $4 \mathrm{~mL}$ fresh culture media.

\section{Cell counting}

1. Gently vortex the cell suspension to ensure homogenous distribution of cells in the centrifuge tube.

2. Using a $10 \mu \mathrm{L}$ pipette, mix $10 \mu \mathrm{L}$ of cell suspension with $10 \mu \mathrm{L}$ of Trypan blue. Gently pipette the mixture up and down four times to ensure complete staining of the outer cell surface with the dye.

3. Count the number of cells using a hemocytometer.

1. First, place a coverslip over the hemocytometer counting area before loading the stained cell suspension.

2. Place the pipette tip containing the cell suspension into the $\mathrm{V}$ groove of the hemocytometer. Gently expel the tip content into the counting slide.

3. Leave the slurry to settle for a couple of minutes before fixing it on the microscope stage for cell counting.

NOTE: To avoid double-counting, only count the cells on the two sides of the large square.

4. Count in cells overlapping the top or the right ruling and avoid those overlapping the bottom or the left ruling.

4. Calculate the total number of cells.

NOTE: Cell number per $\mathrm{ml}=$

$$
\text { Cell count } x \frac{\text { Dilution factor }}{\text { Number of squares }} \times 10^{4}
$$

\section{Collection of fibroblast conditioned media (CM)}

1. Aspirate culture media and wash the LX2 cells three times with PBS.

2. Add $2 \mathrm{~mL}$ of pre-warmed $1 \mathrm{x}$ Trypsin to detach adherent cells from the bottom of the T75 flasks. Incubate the flask at $37^{\circ} \mathrm{C}$ in an incubator for $4 \mathrm{~min}$.

3. Inactivate trypsin by adding $4 \mathrm{~mL}$ of complete culture media. Collect the cell suspension and centrifuge cells at $200 \times g$ for $4 \mathrm{~min}$ at room temperature. Count the cells as per step 3 . 
4. Seed $1 \times 10^{6} \mathrm{LX} 2$ cells in $10 \mathrm{~cm}^{3}$ dishes for $48 \mathrm{~h}$ at $37^{\circ} \mathrm{C}$.

5. Collect the fibroblast $\mathrm{CM}$ after $48 \mathrm{~h}$. Centrifuge at $200 \times \mathrm{g}$ for 4 min at room temperature to pellet any floating cells. Sterile filter the CM using a $0.22 \mu \mathrm{m}$ filter fixed on the bottom of $20 \mathrm{~mL}$ syringes.

6. Collect the supernatant $\mathrm{CM}$. Aliquot the $\mathrm{CM}$ into $2 \mathrm{~mL}$ tubes and store at $-80^{\circ} \mathrm{C}$ for further applications.

NOTE: The solution can be stored for 6 months at $-80^{\circ} \mathrm{C}$.

\section{Validating cell densities for perfect spheroids}

1. Aspirate culture media and wash the $\mathrm{HCC}$ cell lines three times with PBS.

2. Add $2 \mathrm{~mL}$ of pre-warmed $1 \mathrm{x}$ Trypsin to detach adherent cells from the bottom of the $\mathrm{T} 75$ flasks. Incubate at $37^{\circ} \mathrm{C}$ in an incubator for $4 \mathrm{~min}$.

3. Inactivate trypsin by adding $4 \mathrm{~mL}$ of complete culture media. Collect the cell suspension and centrifuge cells at $200 \times g$ for $4 \mathrm{~min}$ at room temperature.

4. Count the cells as per step 3.

5. Pipette different densities from the tumor cell lines $(12000,6000,3000,1500,1000,750,500,250$, and 125 cells) in $20 \mu \mathrm{L}$ of media on the interior surface of a 10 $\mathrm{cm}^{3}$ lid of a Petri dish.

6. Add $10 \mathrm{~mL}$ of sterile PBS to the bottom of the dish to provide humid conditions for the process of spheroid formation.

7. Invert the lid of the $10 \mathrm{~cm}^{3}$ dish to allow the media, including the cell suspension, to hang over a humid environment. Leave the hanging droplets for 3 days.
8. Take images of the spheroids at $50 x$ magnification using an inverted microscope 3 days after hanging the original droplets.

\section{Heterotypic tumor/stromal spheroids}

1. Suspend 1500 Huh7 HCC cells with 1500 COS7 mammalian fibroblast cells (1:1 ratio) in hanging droplets to form spheres. Add $10 \mathrm{~mL}$ of sterile PBS to the bottom of the dish to provide humid conditions for the spheroids.

2. Invert the lid of the $10 \mathrm{~cm}^{3}$ dish to allow the media, including the cell suspension, to hang over a humid environment. Leave the hanging droplets for 3 days.

3. Take images of the spheroids using an inverted microscope from day 3 until day 10 of culture.

1. Put the $10 \mathrm{~cm}^{3}$ dish on the microscope stage. Adjust the magnification of the microscope at 50x for all spheroids.

2. Open the microscope software on the attached computer and adjust its focus to have a clear image of every spheroid. Use the Capture Tool on the microscope software to save the acquired pictures.

\section{Homotypic Hep3B spheroids in LX2 CM}

1. Suspend $3000 \mathrm{Hep} 3 \mathrm{~B}$ HCC cells in the hanging droplets to form spheres. Add $10 \mathrm{~mL}$ of sterile PBS to the bottom of the dish to provide humid conditions for the spheroids.

2. Invert the lid of the $10 \mathrm{~cm}^{3}$ dish to allow the media, including the cell suspension, to hang over a humid environment. Leave the hanging droplets for 3 days.

3. Transfer Hep3B spheroids into $20 \mu \mathrm{L}$ of fresh $\mathrm{CM}$ from LX2 cells in hanging droplets. 
NOTE: Use sterile autoclaved unfiltered $200 \mu \mathrm{L}$ pipette tips in the process of spheroid transfer to avoid any disruption or injury to the formed spheroids. This is also to eliminate any residual cells that remain unattached to the main single spheroid.

1. Use an autoclaved $20 \mu \mathrm{L}$ pipette for the transfer process. Adjust the pipette volume to $2 \mu \mathrm{L}$. Attach the pipette tip to the pipette.

2. Invert the lid of the $10 \mathrm{~cm}^{3}$ dish on which the spheroids were formed. Fix the lid on the stage of a light microscope. Adjust the fine focus of the microscope to make each spheroid visible.

3. Carefully empty the air from the micropipette by pressing the plunger button. Insert the pipette tip in the droplet, including the spheroid, to be transferred. Get very close to the spheroid without touching it with the tip.

4. Gently release the pressure on the plunger button to allow the suction of the spheroid into the micropipette tip in $2 \mu \mathrm{L}$ media.

5. Transfer the spheroid into a new droplet hanging on a new $10 \mathrm{~cm}^{3}$ dish, having new media/conditioned media/treatment.

NOTE: Ensure that all the spheroids are successfully transferred to the new $10 \mathrm{~cm}^{3}$ dish using the light microscope.

4. Take images of the spheroids at 50x magnification using an inverted microscope from the day of transfer (day 3 ) until day 7 of culture in LX2 CM.

\section{Calculation of spheroid volume}

1. Assign a unique numeric identifier for each spheroid so that images from matched spheroids can be captured daily.

2. Analyze images of the growing spheroids using an image analysis software package.

1. Open each spheroid image within the software package. Using the Freehand selection tool and outline each spheroid. From the Analysis dropdown button, select Set Measurement, and then Area. Press OK.

2. Manually draw a circle around each spheroid. Once the sphere is circled, press $\mathbf{C t r l}+\mathbf{M}$ to allow the program to calculate the spheroid area in Pixels. Convert the area of the spheroid into a volume.

NOTE: Volume of spheroidmm3 $=0.09403 \times$

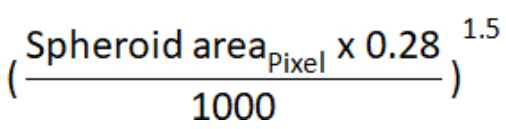

3. Calculate the change in spheroid volume relative to its volume on the first day of image capture.

NOTE: This is to normalize the spheroid volume to the starting volume and improve accuracy given the natural variation in the starting size.

\section{Representative Results}

Cells cultured in a multi-layered 3D format more accurately reflect the complexity of the tumor microenvironment than conventional 2D cultures 24,25 . Previously, many studies have supplemented the spheroids culture media with different mitogens and growth factors ${ }^{26}$ to initiate spheroid formation. In this study, however, the addition of fibroblasts, or their CM, 
provides essential mitogens and growth factors to accelerate spheroid growth.

Figure 1 depicts data from a pilot study in which Huh7 human HCC cells were seeded in a descending seeding density starting from 12,000 down to 125 cells in $20 \mu \mathrm{L}$ fresh media for 3 days. A seeding density of 12,000 cells yielded spheroids with an asymmetric shape, which was not corrected even after halving the cell seeding density. However, spheroids created from 3000 cells appeared more rounded (Figure 1, upper row). Further reduction of cell concentration neither succeeded in forming single spheres (125, 250, 500 celldensity spheres) nor had a regular spherical-like appearance (1000 and 1500 cell-density spheroids). It is worth mentioning that many small spheres were formed at a density of 500 tumor cells; however, only one small spheroid was captured at 50x magnification (Figure 1, upper row). The same optimized protocol was applied to the COS7 primate kidney fibroblast cell line (Figure 1, middle row). Suspending 125, 250, and 500 COS7 fibroblasts in $20 \mu \mathrm{L}$ hanging droplets resulted in one rounded spheroid with multiple smaller spheroids, while higher cell densities $(1000,1500$, and 3000) formed single semi-rounded spheroids. Similar to the Huh7 tumor spheroids, higher cell suspensions (6,000 and 12,000 cells/ sphere) resulted in the formation of irregular cell aggregates, and hence higher cell concentrations were discarded (Figure 1, middle row). Low densities of Huh7/COS7 heterotypic cell suspensions $(125,250$, and 500 cells/sphere) generated a single spheroid with multiple floating or semi-attached spheroids (Figure 1, bottom row). 1000- and 1500-cell heterotypic spheroids were semi-rounded, while a seeding density of 3000 cells (1500 per cell type) gave a rounded 3D spheroid. As noted earlier, higher cell densities resulted in the formation of aggregates rather than well-defined spheroids (Figure 1, bottom row). In conclusion, 3000 cell-density spheroids were rounded, similar to human HCC tumors, and were adapted for further experiments. Culturing the cell suspensions as hanging droplets for 3 days was sufficient to promote spheroid formation.

After optimizing the best cell concentration and time point for spheroid formation in the current context, the proliferative impact of co-culturing tumor and fibroblast cell lines was assessed longitudinally. Figures 2 and Figure $\mathbf{3}$ show Huh7/ COS7 heterotypic spheroids (3000 total cell numbers per spheroid) monitored from day 3 until day 10. Homotypic Huh7 and COS7 spheroids (1500 cells each) served as controls. Starting from day 4 , heterotypic spheroids grew in an ideal round-like shape compared to homotypic spheroids (Figure 2). The initial volume of the heterotypic spheroid was larger than that of each homotypic spheroid. The growth of spheroids was calculated as the change in their volume relative to initial volume at the day of spheroid formation to avoid normal variation in spheroid volume (day 3, Figure 3). Heterotypic spheroids initially showed a rapid growth phase starting from day 4 up to day 7 followed by a slower phase of growth at day 8 (Figures 2, upper row, and Figure 3). The spheroid volume decreased on days 9 and 10, possibly reflecting the depletion of nutrients or a hypoxic core and cell death.

In contrast, homotypic Huh7 and COS7 spheroids grew at a much slower rate (Figure 2, middle and bottom rows; Figure 3). Homotypic spheroids exhibited a relatively static growth curve until the fifth day of culture (two days after spheroid formation). Starting from day 6 , the homotypic spheroids began to show a gradual increase in their growth curve, albeit at a significantly lower rate than that of the heterotypic spheroids (Figure 3). In conclusion, tumor/ fibroblast heterotypic spheroids grow at a higher rate than 
homotypic spheroids suggesting that the direct contact of tumor cells and fibroblasts increases the size of the tumor spheroids.

Finally, to validate the above findings and to study the paracrine impact of mesenchymal cells on the proliferation of HCC spheroids in a liver-related context, 3-day-old homotypic Hep3B HCC spheroids were grown in regular fresh media or CM from LX2 hepatic stellate cells (Figure 4 and Figure 5) for an additional 4 days. At first glance, 3000 Hep3B cells formed perfectly rounded spheroids after 3 days (Figure 4). Hep3B spheroids showed continual proliferation in fresh media from day 3 till day 7 (Figure 4, upper row). The growth rate was enhanced when Hep3B spheroids were maintained in LX2 CM (Figure 4, lower row). This media-dependent conversion in Hep3B spheroid growth exhibited statistical significance from day 4 up to the end of the experiment (Figure 5), suggesting a fibroblast-driven proliferation of tumor spheroids.

In conclusion, this study has successfully modified the existing 3D spheroid cultures to exploit the crosstalk between HCC tumor cells and different fibroblast cell lines and investigate the proliferative significance of this direct and indirect cellular interaction (Figure 6). Further characterization of the formed spheroids is needed to improve how tumor cells interact with the surrounding microenvironment.

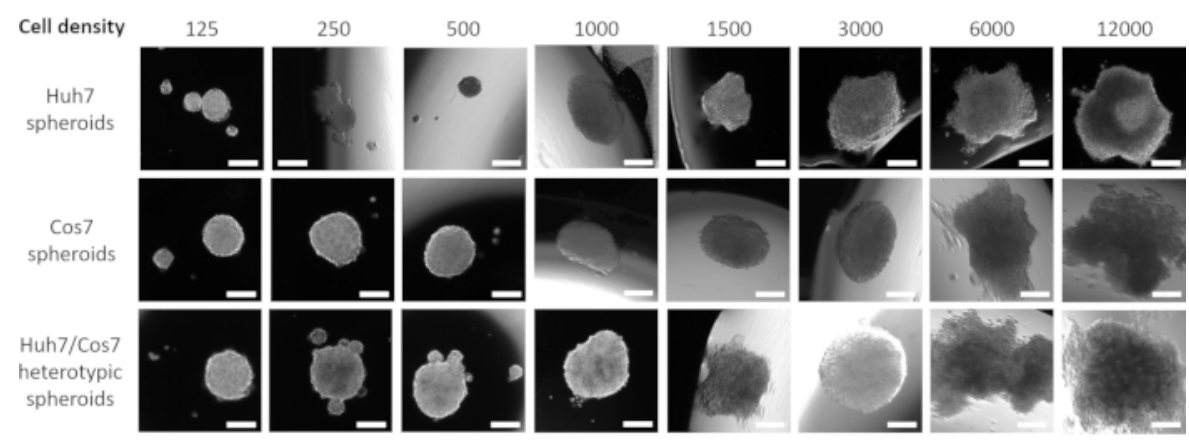

Figure 1: Optimization of the optimal cell density for spheroid formation. Columns represent different cell seeding densities, and rows represent spheroids formed from Huh7 cells (top row), COS7 cells (middle row), and Huh7/COS7 heterotypic cells (bottom row). Images represent spheroids formed after suspending 125, 250, 500, 1000, 1500, 3000, 6000, and 12000 cells (from left to right) in $20 \mu \mathrm{L}$ media for 3 days. The pilot study included two spheroids per condition, and the images were taken at 50x magnification, scale bar $=200 \mu \mathrm{m}$. Please click here to view a larger version of this figure. 


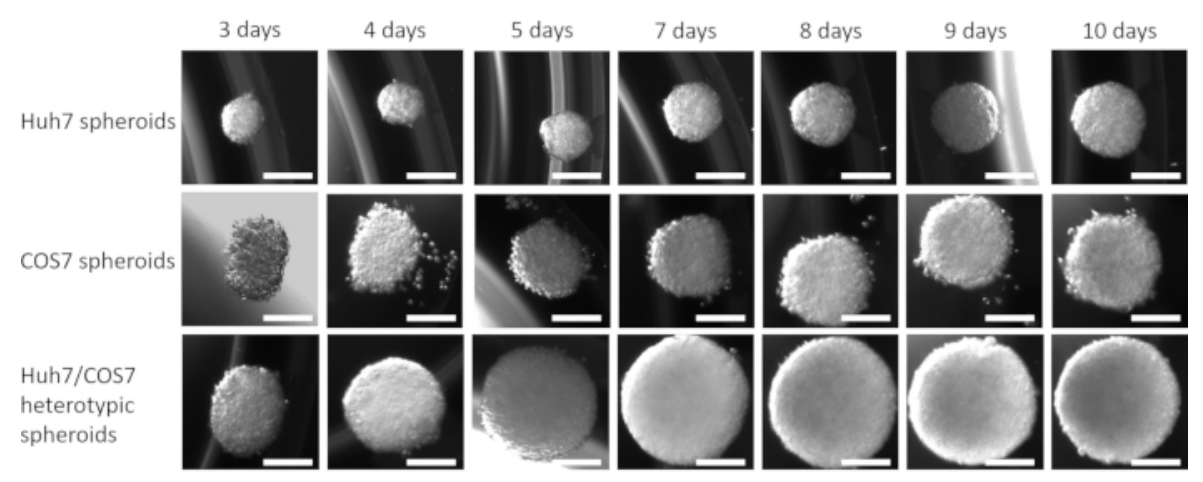

Figure 2: Heterotypic versus homotypic spheroid growth. Columns represent the day at which spheroid images were taken, and rows show representative images for spheroids formed from Huh7 cells (top row), COS7 cells (middle row), and Huh7/COS7 cells (bottom row). The images represent the spheroids formed after suspending 3000 cells from each condition (homotypic Huh7, COS7, or the Huh7/COS7 heterotypic spheroids) of different time points from left to right. The experiment included 10 spheroids per condition, and the images were taken at 50x magnification, scale bar $=200 \mu \mathrm{m}$. Please click here to view a larger version of this figure.

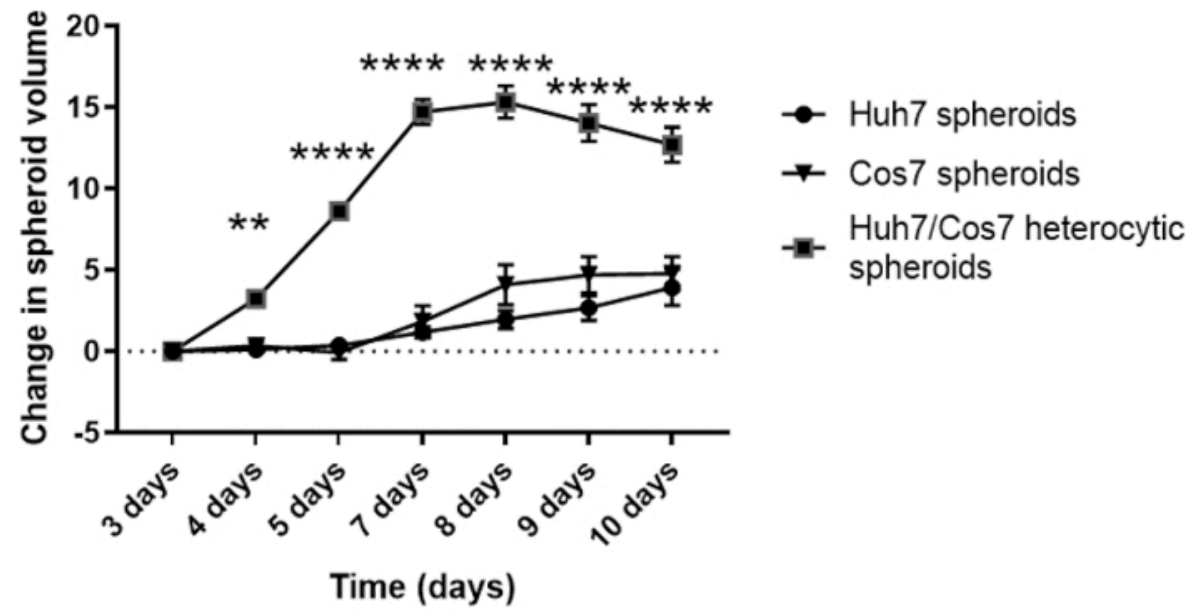

Figure 3: Longitudinal analysis of heterotypic versus homotypic spheroid growth. The graph shows the growth curve of heterotypic Huh7/COS7 spheroids versus homotypic Huh7 and COS7 spheroids. Data are presented as mean \pm s.e.m; $n$ $=10$ independent spheroids. ${ }^{* *} p<0.01 ;{ }^{* * * *} \mathrm{p}<0.001$. Please click here to view a larger version of this figure. 

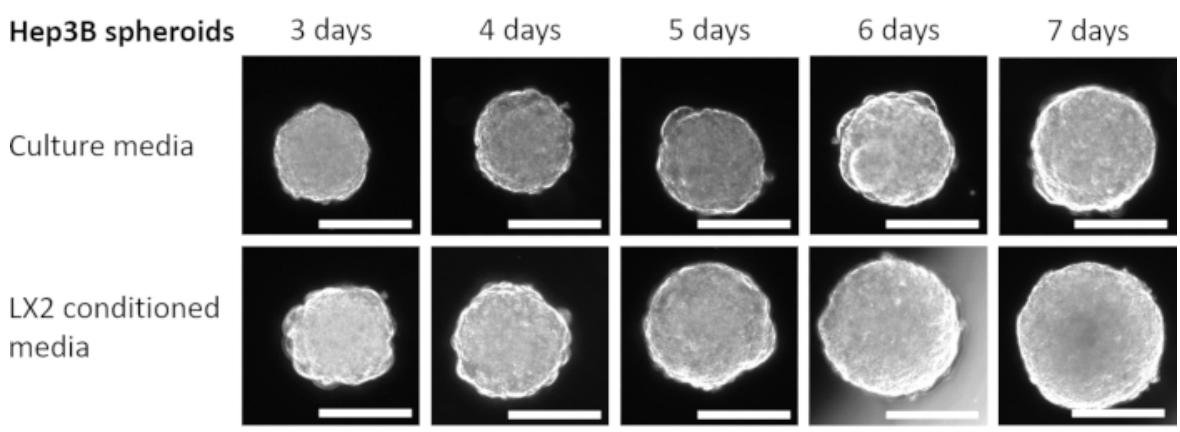

Figure 4: Growth of Homotypic Hep3B spheroids in LX2 CM. Columns represent the day at which spheroid images were captured, and rows show representative images of Hep3B spheroids cultured in fresh DMEM culture media (top row) or LX2 CM (bottom row). The experiment included seven spheroids per condition (fresh media or LX2 CM), and images were taken at 50x magnification, scale bar $=\mathbf{2 0 0} \mu \mathrm{m}$. Please click here to view a larger version of this figure.

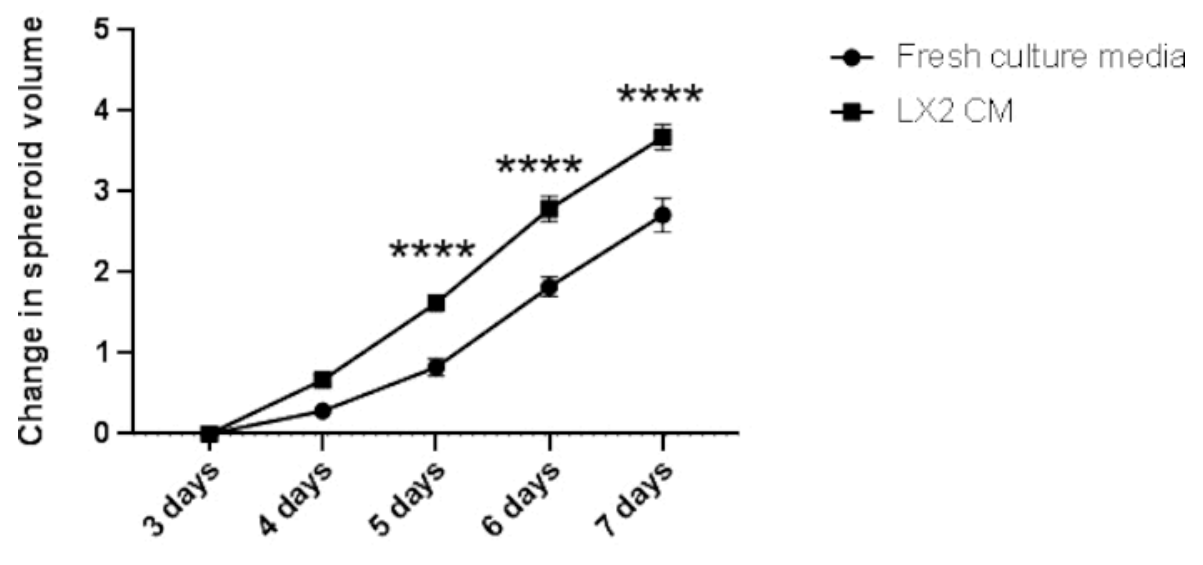

Time (days)

Figure 5: Longitudinal analysis of the homotypic Hep3B spheroid growth. The graph shows the growth curve of Hep3B spheroids in fresh DMEM culture media or LX2 CM. Data are presented as mean \pm s.e.m; $n=7$ independent spheroids. ${ }^{* * * *} \mathrm{p}$ $<0.001$. Please click here to view a larger version of this figure. 


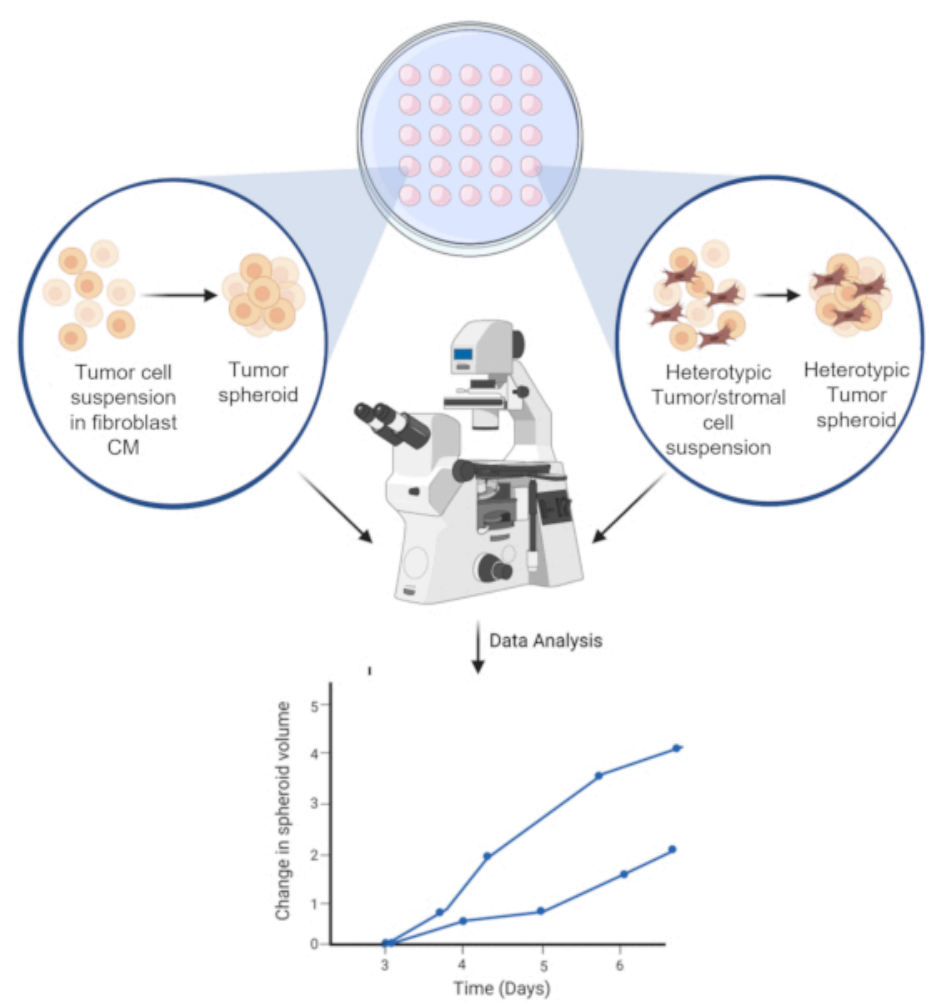

Figure 6: Schematic representation of the spheroid formation process. The cell suspension is pipetted on the inner lid of $10 \mathrm{~cm}^{3}$ Petri-dish. The lid is inverted and kept for 3 days to allow for homotypic or heterotypic spheroid formation. Spheroid images are taken at 50x magnification. The figure is created using a web-based science illustration tool (see Table of Materials). Please click here to view a larger version of this figure.

\section{Discussion}

The context in which the experimental cell lines grow influences their gene expression profile, pathway analysis, and functional criteria. For instance, in breast cancer cells, the coordination between different oncogenic pathways is retained only if cancer cells are grown in $3 \mathrm{D}$ conformation ${ }^{27}$. Deregulated genes in 3D melanoma and breast cancer spheroids, but not in the monolayer cells, are more relevant to the in vivo human tumor 28,29 . For example, $\beta 1$ integrin levels in mammary epithelial cells and tumor counterparts were lower than levels grown in a $2 \mathrm{D}$ format ${ }^{29}$. Furthermore, fibroblasts in $3 D$ structures tend to migrate distinctly in terms of morphology and speed compared to those cultured on plastic $^{30}$. In addition, the mechanical stiffness of the growth substrate activates specific pathways that encourage the malignant transformation of normal epithelial cells via deregulation of the extracellular signal-regulated kinase $(E R K) / R h o$ in epithelial cells ${ }^{31}$. These factors favor the transition from conventional $2 \mathrm{D}$ culture to $3 \mathrm{D}$ spheroid models, as 3D cultures are more in keeping with human disease.

The current study used conventional laboratory tools and supplies to produce a 3D tumor spheroid model. The formed spheroids responded to the proliferation signals coming from 
fibroblasts, as shown by their significantly increased growth. This model belongs to the multicellular tumor spheroids category. There are three other categories of 3D tumor spheroids, including tumorospheres ${ }^{32}$, tissue-derived tumor spheres $^{33,34}$, and organotypic multicellular spheroids ${ }^{35}$. In multicellular tumor spheroids, tumor cells are suspended in low-adhesion conditions to allow them to aggregate together to form spheres without touching the bottom of the culture vesse $^{36}$. Several approaches have been employed to deliver this anchorage-independent technique, ranging from rotating systems, liquid overlay techniques, and uncoated ultra-low attachment U-shaped plates ${ }^{37}$. In HCC, most of the in vitro spheroid cultures use the ultra-low attachment 24- or 96well plates to form rounded spheroids $18,19,20,21,22$. This technique is, however, not cost-effective and doesn't exclude any accidental cell-plastic contact. Other systems use transwells $^{23}$ or liver slices ${ }^{12}$ to produce liver micro-tissues. Using human tissues in translational work is the gold standard but not always available or accessible by many research groups. The current approach benefited from the hanging droplets theory. Cell suspension is added so that the tumor spheroids are formed in an accessible liquid-air interface to form single rounded spheres ${ }^{38}$. Advantages of using this technique are the lack of any cell-plastic contact and the ease of studying the autocrine and paracrine crosstalk between tumor and fibroblast cells. This model was further validated in another study deciphering the importance of the non-parenchymal TREM2 in protecting the liver from HCC development ${ }^{39}$. This work showed that the volume of both Hep3B and PLC/PRF5 spheroids was higher in control LX2 CM versus TREM2overexpressing LX2 CM in a Wnt-dependent manner ${ }^{39}$.

In the current study, fibroblasts were mixed with tumor cells before spheroid formation to investigate the proliferative impact of this co-culture. Others have added fibroblasts, endothelial cells, and immune cells with tumor cell suspension after forming a tumor spheroid to study stromal cell migration into the tumor ${ }^{40,41}$. The current heterotypic spheroid model showed a similar growth pattern to the previously reported models and the original in vivo tumor; spheroids show exponential growth followed by a delayed growth phase, most probably due to the depletion of nutrients and enlargement of the necrotic core ${ }^{42}$. Instead of adding growth factors and mitogens to help spheroid formation, this study used a more physiological approach by having these growth factors from direct contact with fibroblasts or their secretome in the fibroblast CM. It is essential to mention that LX2 cells can be further activated by treating with TGF $\beta 1$ or PDGF ${ }^{43}$. LX2 cells have been treated with $10 \mathrm{ng} / \mathrm{mL}$ TGF $\beta 1$ for $48 \mathrm{~h}$ before removing the media for different experimental settings. LX2 cells were washed three times with PBS (to exclude any direct TGF $\beta 1$ effect), and then fresh media was added for another $24 \mathrm{~h}$ before collecting the CM. The CM collected from TGF $\beta 1$ stimulated LX2 induced the Hep3B spheroids' growth at a higher rate than the CM from control LX2 (data not shown). This flexible system can lend itself to co-cultures of different cancer cells plus/minus fibroblasts, as well as patient-derived lines. It can also offer a medium-throughput screening assay for drugs that could be quickly adopted and inserted into a translational drug discovery pipeline to inform dosing for in vivo studies.

A limitation of the current study is the use of the immortalized cell lines in spheroid formation rather than freshly isolated HCC tumor cells and cancer-associated fibroblasts. Another limitation is comparing the homotypic Hep3B spheroid's growth between CM from LX2 and in fresh media rather than CM from other non-fibroblast cell lines. The latter limitation could be addressed by the genetic modification of a gene of interest in the fibroblasts followed by applying the $\mathrm{CM}$ 
from wild type versus genetically engineered fibroblast to homotypic spheroids.

\section{Disclosures}

FO is a director and shareholder of Fibrofind limited.

\section{Acknowledgments}

MYWZ is funded by the The Secretary of State for Business, Energy and Industrial Strategy and the Newton Prize 2020 as a part of the UK's Official Development Assistance "ODA" and Newton fund. SS is supported by Cancer Research UK Advanced Clinician Scientist fellowship C53575/A29959. SS, FO, and HR receive funding as part of HUNTER, funded through a partnership between Cancer Research UK, Fondazione AIRC, and Fundacion Cientifica de la Asociacion Espanola Contra el Cancer.

\section{References}

1. Ferlay, J. et al. Cancer statistics for the year 2020: an overview. International Journal of Cancer. (2021).

2. Llovet, J. M. et al. Hepatocellular carcinoma. Nature Reviews Disease Primers. 7 (1), 6 (2021).

3. Reeves, H. L., Zaki, M. Y., Day, C. P. Hepatocellular carcinoma in obesity, Type 2 diabetes, and NAFLD. Digestive Diseases \& Sciences. 61 (5), 1234-1245 (2016).

4. Forner, A., Reig, M., Bruix, J. Hepatocellular carcinoma. Lancet. 391 (10127), 1301-1314 (2018).

5. Reig, M. et al. Diagnosis and treatment of hepatocellular carcinoma. Update of the consensus document of the AEEH, AEC, SEOM, SERAM, SERVEI, and SETH. Medicina Clinica (Barc). 156 (9), 463.e1-463.e30 (2021).
6. Pfister, D. et al. NASH limits anti-tumour surveillance in immunotherapy-treated HCC. Nature. 592 (7854), 450-456 (2021).

7. Llovet, J. M. et al. Trial design and endpoints in hepatocellular carcinoma: AASLD consensus conference. Hepatology. 73 Suppl 1, 158-191 (2021).

8. Llovet, J. M., Hernandez-Gea, V. Hepatocellular carcinoma: reasons for phase III failure and novel perspectives on trial design. Clinical Cancer Research. 20 (8), 2072-2079 (2014).

9. Brown, Z. J., Heinrich, B., Greten, T. F. Mouse models of hepatocellular carcinoma: an overview and highlights for immunotherapy research. Nature Reviews Gastroenterology and Hepatology. 15 (9), 536-554 (2018).

10. Nikolic, M., Sustersic, T., Filipovic, N. In vitro models and on-chip systems: Biomaterial interaction studies with tissues generated using lung epithelial and liver metabolic cell lines. Frontiers in Bioengineering and Biotechnology. 6, 120 (2018).

11. Clark, A. M., Ma, B., Taylor, D. L., Griffith, L., Wells, A. Liver metastases: Microenvironments and ex-vivo models. Experimental Biology and Medicine. 241 (15), 1639-1652 (2016).

12. Paish, H. L. et al. A bioreactor technology for modeling fibrosis in human and rodent precision-cut liver slices. Hepatology. 70 (4), 1377-1391 (2019).

13. Brassard-Jollive, N., Monnot, C., Muller, L., Germain, S. In vitro 3D systems to model tumor angiogenesis and interactions with stromal cells. Frontiers in Cell and Developmental Biology. 8, 594903 (2020). 
14. Pampaloni, F., Reynaud, E. G., Stelzer, E. H. The third dimension bridges the gap between cell culture and live tissue. Nature Reviews Molecular Cell Biology. 8 (10), 839-845 (2007).

15. Kleinman, H. K., Philp, D., Hoffman, M. P. Role of the extracellular matrix in morphogenesis. Current Opinion in Biotechnology. 14 (5), 526-532 (2003).

16. Bissell, M. J., Radisky, D. C., Rizki, A., Weaver, V. M., Petersen, O. W. The organizing principle: microenvironmental influences in the normal and malignant breast. Differentiation. 70 (9-10), 537-546 (2002).

17. Ronnov-Jessen, L., Petersen, O. W., Koteliansky, V. E., Bissell, M. J. The origin of the myofibroblasts in breast cancer. Recapitulation of tumor environment in culture unravels diversity and implicates converted fibroblasts and recruited smooth muscle cells. Journal of Clinical Investigations. 95 (2), 859-873 (1995).

18. Shao, $H$. et al. A novel stromal fibroblast-modulated 3D tumor spheroid model for studying tumor-stroma interaction and drug discovery. Journal of Visualized Experiments: JoVE. 156, e60660 (2020).

19. Song, Y. et al. Activated hepatic stellate cells play pivotal roles in hepatocellular carcinoma cell chemoresistance and migration in multicellular tumor spheroids. Scientific Reports. 636750 (2016).

20. Pingitore, P. et al. Human multilineage 3D spheroids as a model of liver steatosis and fibrosis. International Journal of Molecular Sciences. 20 (7) (2019).

21. Kozyra, M. et al. Human hepatic 3D spheroids as a model for steatosis and insulin resistance. Scientific Reports. 8 (1), 14297 (2018).
22. Khawar, I. A. et al. Three dimensional mixed-cell spheroids mimic stroma-mediated chemoresistance and invasive migration in hepatocellular carcinoma. Neoplasia. 20 (8), 800-812 (2018).

23. Feaver, R. E. et al. Development of an in vitro human liver system for interrogating non-alcoholic steatohepatitis. Journal of Clinical Investigations Insight. 1 (20), e90954 (2016).

24. Wartenberg, $M$. et al. Regulation of the multidrug resistance transporter $\mathrm{P}$-glycoprotein in multicellular tumor spheroids by hypoxia-inducible factor (HIF-1) and reactive oxygen species. Federation of American Societies for Experimental Biology Journal. 17 (3), 503-505 (2003).

25. Chen, R. et al. Screening candidate metastasisassociated genes in three-dimensional HCC spheroids with different metastasis potential. International Journal of Clinical Experimental Pathology. 7 (5), 2527-2535 (2014).

26. Venkataraman, G. et al. Preferential self-association of basic fibroblast growth factor is stabilized by heparin during receptor dimerization and activation. Proceedings of the National Academy of Sciences of the United States of America. 93 (2), 845-850 (1996).

27. Bissell, M. J. et al. Tissue structure, nuclear organization, and gene expression in normal and malignant breast. Cancer Research. 59 (7 Suppl), 1757-1763s; discussion 1763s-1764s (1999).

28. Ghosh, S. et al. Three-dimensional culture of melanoma cells profoundly affects gene expression profile: a high density oligonucleotide array study. Journal of Cellular Physiology. 204 (2), 522-531 (2005). 
29. Delcommenne, M., Streuli, C. H. Control of integrin expression by extracellular matrix. Journal of Biological Chemistry. 270 (45), 26794-26801 (1995).

30. Meshel, A. S., Wei, Q., Adelstein, R. S., Sheetz, M. P. Basic mechanism of three-dimensional collagen fibre transport by fibroblasts. Nature Cell Biology. 7 (2), 157-164 (2005).

31. Paszek, M. J. et al. Tensional homeostasis and the malignant phenotype. Cancer Cell. 8 (3), 241-254 (2005).

32. Yu, M. et al. Cancer therapy. Ex vivo culture of circulating breast tumor cells for individualized testing of drug susceptibility. Science. 345 (6193), 216-220 (2014).

33. Weiswald, L. B. et al. Newly characterised ex vivo colospheres as a three-dimensional colon cancer cell model of tumour aggressiveness. British Journal of Cancer. 101 (3), 473-482 (2009).

34. Kondo, J. et al. Retaining cell-cell contact enables preparation and culture of spheroids composed of pure primary cancer cells from colorectal cancer. Proceedings of the National Academy of Sciences of the United States of America. 108 (15), 6235-6240 (2011).

35. Rajcevic, U. et al. Colorectal cancer derived organotypic spheroids maintain essential tissue characteristics but adapt their metabolism in culture. Proteome Sciences. 12, 39 (2014).

36. Friedrich, J., Seidel, C., Ebner, R., Kunz-Schughart, L. A. Spheroid-based drug screen: considerations and practical approach. Nature Protocols. 4 (3), 309-324 (2009).

37. Friedrich, J., Ebner, R., Kunz-Schughart, L. A. Experimental anti-tumor therapy in 3-D: spheroids--old hat or new challenge? International Journal of Radiation Biology. 83 (11-12), 849-871 (2007).

38. Kelm, J. M., Timmins, N. E., Brown, C. J., Fussenegger, M., Nielsen, L. K. Method for generation of homogeneous multicellular tumor spheroids applicable to a wide variety of cell types. Biotechnology \& Bioengineering. 83 (2), 173-180 (2003).

39. Esparza-Baquer, A. et al. TREM-2 defends the liver against hepatocellular carcinoma through multifactorial protective mechanisms. Gut. 70 (7), 1345-1361 (2021).

40. Dangles-Marie, V. et al. A three-dimensional tumor cell defect in activating autologous CTLs is associated with inefficient antigen presentation correlated with heat shock protein-70 down-regulation. Cancer Research. 63 (13), 3682-3687 (2003).

41. Timmins, N. E., Dietmair, S., Nielsen, L. K. Hangingdrop multicellular spheroids as a model of tumour angiogenesis. Angiogenesis. 7 (2), 97-103 (2004).

42. Mayer, B. et al. Multicellular gastric cancer spheroids recapitulate growth pattern and differentiation phenotype of human gastric carcinomas. Gastroenterology. 121 (4), 839-852 (2001).

43. Xu, L. et al. Human hepatic stellate cell lines, LX-1 and LX-2: new tools for analysis of hepatic fibrosis. Gut. $\mathbf{5 4}$ (1), 142-151 (2005). 